Physics

Physics Research Publications

\title{
Laboratory test of Newton's second law for small accelerations
}
J. H. Gundlach*
S. Schlamminger ${ }^{\dagger}$
K. Y. Choi**
B. A. Woodahl ${ }^{\dagger \dagger}$
J. J. Coy
E. Fischbach ${ }^{\S}$

C. D. Spitzer ${ }^{\ddagger}$

$\dagger$
$\ddagger$
$* *$
$\dagger \dagger$
$\ddagger$
$\S$

This paper is posted at Purdue e-Pubs.

http://docs.lib.purdue.edu/physics_articles/870 


\title{
Laboratory Test of Newton's Second Law for Small Accelerations
}

\author{
J. H. Gundlach, S. Schlamminger, C. D. Spitzer, and K.-Y. Choi \\ Center for Experimental Nuclear Physics and Astrophysics, University of Washington, Seattle, Washington 98195, USA
}

B. A. Woodahl

Physics Department, Indiana University-Purdue University, Indianapolis, Indiana 46202, USA

J. J. Coy

Earth and Space Science Department, Saint Joseph's College, Rensselaer, Indiana 47978, USA

E. Fischbach

Physics Department, Purdue University, West Lafayette, Indiana 47907, USA

(Received 12 February 2007; published 13 April 2007)

\begin{abstract}
We have tested the proportionality of force and acceleration in Newton's second law, $F=m a$, in the limit of small forces and accelerations. Our tests reach well below the acceleration scales relevant to understanding several current astrophysical puzzles such as the flatness of galactic rotation curves, the Pioneer anomaly, and the Hubble acceleration. We find good agreement with Newton's second law at accelerations as small as $5 \times 10^{-14} \mathrm{~m} / \mathrm{s}^{2}$.
\end{abstract}

DOI: 10.1103/PhysRevLett.98.150801

PACS numbers: 06.30.Gv, 04.80.Cc

Newton's second law is the equation of motion defining the field of dynamics. In its nonrelativistic form, $\vec{F}=m \vec{a}$ is perhaps the most famous and most often used equation of physics. Together with its relativistic and quantum mechanical variants, this law is implicitly tested in many applications and experiments, and its validity is simply assumed at all acceleration scales. Any deviation from $\vec{F}=m \vec{a}$ would have profound consequences as it would imply a violation of crucial conservation laws such as energy and momentum in their conventional definition. At very small accelerations a deviation from Newton's second law could remain hidden in most laboratory scale experiments, but might appear in astrophysical and cosmological observations.

One observed fact is the flatness of galactic rotation curves. The tangential velocity of stars measured as a function of distance from the galactic center rises first and flattens for larger distances. Newton's second law together with the gravitational effect of known matter predicts a decrease in the velocities for larger distances, and dark matter has been introduced to resolve this discrepancy [1]. Alternatively, Milgrom discovered that Newton's second law can be modified with a single additional parameter $a_{0}$ to describe the measured galactic rotation curves extremely well without invoking dark matter [2,3]. While Milgrom's full formalism MOND (modified Newtonian dynamics) is untestable in the laboratory, since it requires the absence of accelerations in all directions, a modification of Newtonian dynamics provides a simple explanation of the galactic rotation curves. Milgrom suggested that Newton's second law would smoothly transition from $F \propto a$ to $F \propto a^{2} / a_{0}$ at $a \approx a_{0}$. Hence, for $a \ll$ $a_{0}$ a force would yield a larger acceleration as compared to standard Newtonian dynamics. The functional form of the transition between the two regimes is not specified. A smooth transition can be obtained by multiplying the right side of $\vec{F}=m \vec{a}$ by $\mu\left(a / a_{0}\right)=a / a_{0}\left(1+a^{2} / a_{0}^{2}\right)^{-1 / 2}$, so that for $a \gg a_{0}$ the function $\mu \simeq 1$ and standard Newtonian mechanics is recovered. The characteristic acceleration $a_{0}$ was determined from fits [4] to galactic rotation curves to be $a_{0} \approx 1.2 \times 10^{-10} \mathrm{~m} / \mathrm{s}^{2}$.

Further testing of Newton's second law is motivated by the Pioneer anomaly. Doppler-tracking data of the Pioneer 10 and 11 spacecraft shows an unmodeled acceleration at distances $>15$ AU of $a_{0} \approx 9 \times 10^{-10} \mathrm{~m} / \mathrm{s}^{2}$ roughly pointing towards the Sun [5]. Exhaustive efforts have been undertaken to find a conventional explanation for this effect, so far without success. It is also interesting to note that the Hubble acceleration $a_{H}=c H \approx 7 \times 10^{-10} \mathrm{~m} / \mathrm{s}^{2}$ provides a natural acceleration scale, with $H$ being the Hubble constant. Furthermore, it is also conceivable that a violation of Newton's second law could play a role in explaining the acceleration of the Universe at large distances, which is usually attributed to dark energy.

Here we report the results of a laboratory experiment to test Newton's second law using a torsion pendulum. Our system is significantly different from that used in an earlier experiment by Abramovici and Vager [6], who interferometrically measured the acceleration of a pendulum mass in response to an applied electric field. Abramovici and Vager found agreement with Newton's second law at accelerations as small as $3 \times 10^{-11} \mathrm{~m} / \mathrm{s}^{2}$. In our experiment we utilize the fact that as a torsion pendulum passes through equilibrium its acceleration (relative to the laboratory) is zero. If the torsional amplitude is small, the time spent experiencing small accelerations and small forces 
can be made sufficiently long, so that the free torsional period would be altered. Therefore measuring the pendulum's period as a function of amplitude is a direct test of Newton's second law.

In the simplified case of a pendulum where all the mass is concentrated at one radius, as for a hollow cylinder with radius $r$, a MOND-like free equation of motion relates the acceleration $a$ to the inertial torque on the pendulum $\tau$ :

$$
\tau\left(I, a, a_{0}\right)=\frac{I a}{r} \mu\left(a / a_{0}\right),
$$

where $I$ is the moment of inertia of the torsion pendulum. For a pendulum with an arbitrary mass distribution given by the radial mass density $\rho(r)$ the equation of motion implied by (1) is then

$$
\left(\int \mu\left(|r \ddot{\theta}| / a_{0}\right) \rho(r) r^{2} d V\right) \ddot{\theta}+\kappa \theta=0 .
$$

Here $\theta$ is the angular displacement of the torsion pendulum, $r \ddot{\theta}=a$ is the acceleration, $\kappa$ is the fiber's torsional constant, and the overdots denote time derivatives. Figure 1 shows a numerical solution of Eq. (2) in comparison with a measured trace. The simulation demonstrates that in the MOND-like regime the period of oscillations is shorter than predicted by Newton's second law, just as is the case for stars far out in the galaxy which experience similarly small forces.

The instrument used for this test is the University of Washington's equivalence principle apparatus, which consists of a torsion balance installed on an air bearing turn-

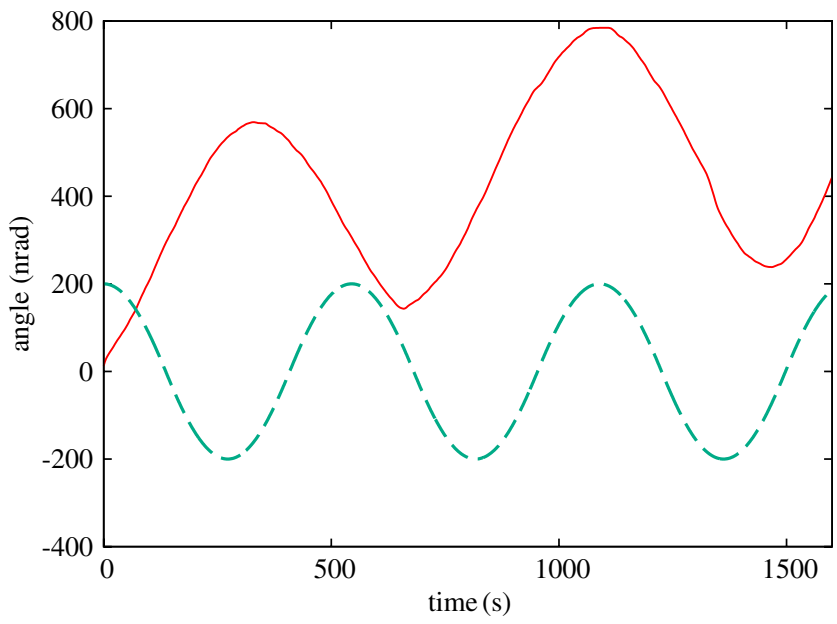

FIG. 1 (color online). The solid line shows a typical measurement of the angular position of the torsion pendulum. The upward drift in the data is due to a slow unwinding of the torsion fiber. The noise in the measured trace is due to Brownian excitation and readout noise. For purposes of comparison the simulated motion of the pendulum in a MOND-like regime with $a_{0}=1.0 \times 10^{-12} \mathrm{~m} / \mathrm{s}^{2}$ is plotted with a dashed line. Note that the MOND-like dynamics would result in a much shorter period of oscillation. table. The pendulum is suspended from a $1.07 \mathrm{~m}$ long, $20 \mu \mathrm{m}$ thick tungsten wire, with $\kappa=2.36 \times$ $10^{-9} \mathrm{~N} \mathrm{~m} / \mathrm{rad}$. The natural period of the pendulum is $795 \mathrm{~s}$ and the amplitude decay time is 15 days, corresponding to a quality factor, $Q$, of approximately 5000. The pendulum is suspended inside a vacuum chamber at a pressure of $10^{-5} \mathrm{~Pa}$, maintained by an ion pump.

Before each measurement the torsion pendulum was actively damped to very small amplitudes by rotating the turntable on which the torsion balance is installed. The turntable was then kept stationary and the torsional period was measured as a function of amplitude. The damping was necessary since the pendulum gained on average a torsional amplitude of about $60 \mathrm{nrad}$ in approximately one period, mostly due to Brownian excitation in the fiber. Once the pendulum gained a substantial amplitude the data taking was stopped. The acquired torsional amplitude was removed by instantly rotating the turntable so that no energy remained stored in the twist of the fiber, and after that the data taking was resumed. Over a period of 20 days we operated the pendulum at various amplitudes ranging from approximately $13 \mathrm{nrad}$ to $19 \mu \mathrm{rad}$. A typical data trace is shown as the solid line in Fig. 1. The linear drift in the data is caused by a slow and constant relaxation of the torsion fiber. The noise at low frequencies $(\leq 10 \mathrm{mHz})$ is predominantly caused by Brownian excitation, while at higher frequencies it is dominated by the optical readout.

The angular position of the pendulum was measured with an autocollimator and was recorded every $2.74 \mathrm{~s}$. The data analysis began by averaging every 20 data points. The data were then prefiltered with an algorithm that identified large instantaneous changes in angular momentum (spikes). These impulses were often related to pressure bursts of the ion pump, and sections containing such spikes were eliminated from the data, leading to 326 spike free data sections of various lengths. We used the zero crossings of the second derivatives of the data to find the pendulum's equilibrium position and hence detect the linear drift which was then subtracted from the raw (nonaveraged) data. To each section of this drift corrected data we fit a variable frequency sinusoid using a standard Levenberg-Marquardt technique [7]. For each section we obtain the measured frequency and amplitude and their corresponding uncertainties.

To convert the measured angular variables to linear variables we used the mean effective radius $r_{e}=0.023 \mathrm{~m}$, calculated from the pendulum's mass distribution. Combining this radius with the measured amplitudes $A$ and frequencies $\omega /(2 \pi)$, we calculate the maximum force using Hooke's law, $F=A \kappa / r_{e}$, and the maximum acceleration for the torsion pendulum, which occurs at the turning points and is given by $a=A r_{e} \omega^{2}$. Figure 2 shows the inferred accelerations versus force for all fitted data sections. Also shown is the deviation in acceleration and force from $F=m a$ with $m$ as the only fit parameter. Our data 


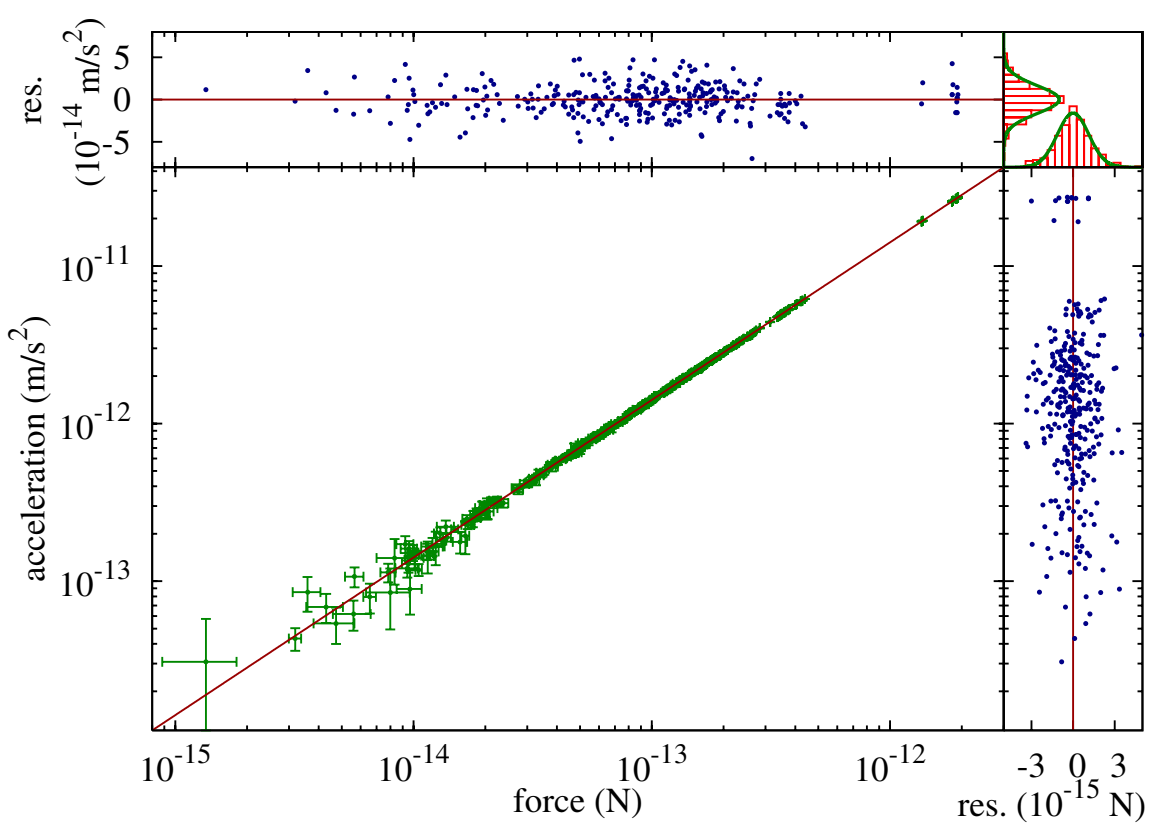

FIG. 2 (color online). The measured force versus the measured acceleration. The solid line is the best fit for acceleration, $a$ being exactly proportional to force $F$. Our data agree very well with the curve. The insets on the right and top of the main graph give the residuals of the data to the fitted line.

indicate that force and acceleration remain proportional down to accelerations as small as $5 \times 10^{-14} \mathrm{~m} / \mathrm{s}^{2}$.

We conducted numerous checks for bias and systematic uncertainties in our data analysis. We performed an independent alternative data analysis in which the data were divided into 1200 and $2000 \mathrm{~s}$ long sections irrespective of the spikes, which were not removed. In this analysis drift was accounted for by introducing a linear and quadratic term in the fit. Data sections that exhibited exceedingly large $\chi^{2}$ were eliminated. This data analysis also produced good agreement with Newton's law, but had slightly larger uncertainties.

Our main data analysis was tested with two sets of simulated data. The first set contained a realistic spectral noise distribution of a harmonic oscillator and white readout noise. The frequencies were recovered properly for amplitudes comparable to the measured traces, as well as for smaller amplitudes. The uncertainties and the $\chi^{2}$ behaved similarly to the corresponding quantities in the measured data. The second set contained simulated MONDlike data with a range of $a_{0}$ from $10^{-16}-10^{-9} \mathrm{~m} / \mathrm{s}^{2}$. All the accelerations were recovered within uncertainties.

In summary, we have found no deviation from the proportionality in Newton's second law down to accelerations of $5 \times 10^{-14} \mathrm{~m} / \mathrm{s}^{2}$, which is approximately 1000 times smaller than the previous 1986 test. In fact, all our data points were measured at accelerations that were smaller than the smallest acceleration in Ref. [6]. Our ability to measure at even smaller accelerations is complicated by the thermal excitation of the torsion pendulum. Since we find good agreement with $F=m a$ it is unlikely that a possible violation of Hooke's law would exactly hide a violation of $F=m a$. Our torsion fiber is expected to adhere ideally to Hooke's law because it is operated far from its inelastic limit and also because the oscillator has a high $Q$. Our test does not invalidate MOND directly, since MOND requires that the measurement must be carried out in the absence of any other larger accelerations, such as those due to the earth and our solar system. However, our results constrain any theoretical formalism seeking to derive MOND from fundamental principles [8] by requiring that formalism to reproduce $F=m a$ under laboratory conditions similar to those in our experiment.

For a future measurement we plan to test $F_{g}=m a$ at small accelerations, where $F_{g}$ is a gravitational force as in the galactic dynamics.

The work at the University of Washington is supported by the NSF under Grant No. NSF PHY-0355012 and E. F. is supported in part by the U.S. Department of Energy under Contract No. DE-AC02-76ER071428.

[1] Y. Sofue and V. Rubin, Annu. Rev. Astron. Astrophys. 39, 137 (2001).

[2] M. Milgrom, Astrophys. J. 270, 365 (1983).

[3] M. Milgrom, Astrophys. J. 270, 371 (1983).

[4] R.H. Sanders and S.S. McGaugh, Annu. Rev. Astron. Astrophys. 40, 263 (2002).

[5] J. D. Anderson et al., Phys. Rev. D 65, 082004 (2002).

[6] A. Abramovici and Z. Vager, Phys. Rev. D 34, 3240 (1986).

[7] W.H. Press, S. A. Teukolsky, W. T. Vetterling, and B.P. Flannery, Numerical Recipes in C: The Art of Scientific Computing (Cambridge University Press, Cambridge, England, 2002), 2nd ed.

[8] J. D. Bekenstein, Phys. Rev. D 70, 083509 (2004). 